\title{
Withering Internship Training In India
}

\author{
Anand Kumar
}

Published online: 6 April 2011

(C) Association of Surgeons of India 2011

Why we need to redress this issue? What are the objectives of internship training? Why it is essential to have internship? Why internship training is been taken casually? Who is responsible for this grave situation? These are few issues which take me to deep thoughts as a medical educationist and force me to analyse, visualise and conceptualise the issues put forward as withering internship training in the country.

On one side we are trying to reform the higher education because of the increasing knowledge, technology and its application as well as need based education while on other side we are forced to be critical about our existing practices with reference to acquiring skills following MBBS degree. Internship is an integral part of bachelor's degree and without this a student is not certified as medical graduate. Nearly $20 \%$ of the period of complete curriculum is the internship during which one is expected to perform oneself the basic skills required for a basic doctor in clinical specialities. This training is also required for the fulfilment to obtain the registration to practice medicine. These objectives in itself prove the importance of this very component of the curriculum. There is a syllabus of every subject, a student learns during MBBS which is evaluated before pass certificate is awarded. Unfortunately internship neither has a syllabus nor any evaluation. It is by convention and departmental generosity that the students

\footnotetext{
A. $\operatorname{Kumar}(\square)$

Department of General Surgery, Institute of Medical Sciences, Banaras Hindu University,

Varanasi 221005 , India

e-mail: profanandkumar52@gmail.com

A. Kumar

B-143, Brij Enclave Colony, Sunderpur,

Varanasi, Uttar Pradesh, India
}

get the certificate of having completed the internship and the degree is awarded based on the certificate so issued. Though one is supposed to acquire all expected skills including emergency medicine but the approach to this important event is in a state of shock.

Medical Council of India has designed specific objectives of the internship training program for each speciality. How much and to what extent we are able to achieve the defined goals questions the validity of such objectives? Any laid down protocol needs evaluation, validation and implementation. Unfortunately all the above parameters required are practically to decimal level. The Medical Council of India has made a recent amendment of giving a score based on few broad questions to obtain a satisfactory completion certificate but this is not implemented uniformly all over the country. The problem is compounded because at times some departments are not geared to satisfactorily monitor and regulate the internship training program. The students have the opportunity to complete the training period in hospitals which do not have any experience for training.

This is completely being ignored and considered as least important aptly from trainees as well as trainers. Having narrated the reasons for redressal of problems one is again forced to analyse why are we across the roads in meeting our objectives of internship. The period instead of acquiring skills in patients approach towards being the basic doctors is spent as a paid preparatory period for their future i.e. post graduate entrance examination. The latitude given by the institutions and various departments has practically thrown the internship as a vehicle without brake on a crowded street. One can imagine the fate arising out of this situation where the graduate degree is being awarded without acquiring the required basic skills. Instead of being progressive we are regressing by one year. Those who join as first year resident do not know the alphabets of required 
skills and start working in the subject and they lack the basics of other subjects. The so called basic doctor supposed to be capable and competent to serve the public at large are unskilled and is a matter of great concern. There is increasing need of doctors in rural India for common medical ailments. The internship which is practically inhouse in majority of the situation and only certificate based could be exploited as compulsory for six months in rural set-up. It may be argued that that they get training in $\mathrm{CHC} /$ PHC for a period of 3 months seems perfect but this six month should be additional before they get admission to post graduate course.

Pursuance of post-graduation training, i.e. specializing, is a priority for passing medical graduates because of the prestige and earning potential associated with it. The consulting fees and pay packets of specialists are routinely higher than general practitioners. Unfortunately, the majority of interns do not actively participate in the internship period for the reasons that in many states of India, the graduate medical doctors have to take a written examination for pursuance of post-graduate studies. The numbers of positions available for post-graduation training programs, i.e. specialization in any of the medical or surgical branches are few compared to the number of passing medical graduates. Many students do not take internship period seriously but would rather study for their pre-PG examination. Some of them succeed after one or more attempts. However, the limited number of PG seats ensures that majority of doctors remain as basic medical graduates who are otherwise unskilled due to inadequate internship training.

In conclusion, the objective of providing an excellent training during internship period to improve the training of medical graduates in India cannot be achieved unless the interns actively participate in their scheduled activities as per training program. It has been aptly stated that internship is one of the weakest links of the teaching program which otherwise should be robust. In fact internship is considered by some as a vacation period or paid holidays and such myths have to be condemned. Graduating doctors enter into medical practice as private entrepreneurs, on salaried posts or proceed for specialization studies. This practice has grave consequences for doctors, whether generalists, i.e. basic medical graduates or specialists, as they have largely missed out on the basic crucial training component of internship. Thus there is an urgent need to redress this issue in order to make internship effective, fruitful and commensurate with the needs of the society. This will be a bench mark approach towards the concept of a "five-star doctor" who possess excellent skills as a care provider, decision maker, communicator, community leader and manager. 